Aim To determine influence of oxygen support on diameter and flow rate through the pulmonary artery in preterm infants by echocardiography.

Methods The study was conducted in tertiary health institution (Pediatric Clinic, KCU Sarajevo), the Neonatal intensive care unit between November 2011th and February 2012th. Examinees were divided into three groups, depending on the type of oxygen support: infants on conventional mechanical ventilation, CPAP by continuous positive pressure and indirect oxygen. It was performed echocardiography measuring of pulmonary artery dimensions and the maximum flow rate. Standard echocardiography methods were used: One-dimensional M mode and Two-dimensional Doppler.

Results 30 patients age $<35$ weeks were divided into three groups.

Mean body weight was $1066 \pm 150.5 \mathrm{~g} ; 1280 \pm 115.95 \mathrm{~g}$ and $2720 \pm 420.92 \mathrm{~g}$.

Mean gestational age is: $28.7 \pm 1.78 ; 30.3 \pm 0.95$ and $33.90 \pm 0.99$.

The mean diameter of the main branch of the pulmonary artery is: $5.64 \pm 0.6 ; 6.16 \pm 0.37$ and $8.5 \mathrm{~mm} \pm 1.06$.

The mean maximum flow rate through the main branch of the pulmonary artery $(\mathrm{m} / \mathrm{s}): 1.48 \pm 0.74 ; 1.58 \pm 0.24$ and $1.72 \pm 0.18$.

Conclusion By comparison of dimension values and flow rate through the pulmonary artery we have proved that type of oxygen support has no influence on the diameter and flow speed through the pulmonary artery in preterm infants $<35$ weeks of gestation.

\section{ISOLATED AORTIC COARCTATION SEEN IN THE CHILDREN IN REPUBLIC OF KOSOVA A 12YEARS SINGLE CENTRE EXPERIENCE}

doi:10.1136/archdischild-2012-302724.1137

${ }^{1} \mathrm{R}$ Bejiqi, ${ }^{2} \mathrm{R}$ Retkoceri, ${ }^{2} \mathrm{~N}$ Zeka, ${ }^{3} \mathrm{H}$ Bejiqi, ${ }^{2} \mathrm{M}$ Kelmendi, ${ }^{4} \mathrm{~A}$ Retkoceri, ${ }^{3} \mathrm{~S}$ Surdulli. 'Pediatric Clinic, University Children's Hospital, Clinical Center of Kosovo; ${ }^{2}$ Pediatric Clinic, University Clinical Center of Kosova; ${ }^{3}$ Main Center of Family Medicine; ${ }^{4}$ Main Center of Family Medicine, Prishtina, Kosovo

Introduction Aortic coarctation (CoA) accounts for 6 to $8 \%$ of live births with congenital heart disease and it represents a spectrum of lesions, generally encompassing variable degrees of tubular hypoplasia along with additionally stenotic areas within the aortic arch.

Objective Aim of this presentation is to describe pathoanatomical presentation, echocardiographic diagnosis, treatment and outcome of children with isolated CoA.

Methods Retrospectively we analyzed medical records and echocardiograms of 62 children with CoA during the past 11 years.

Results Patients ( $n=61,38$ male or $62 \%$ ) diagnosed with a median age of 14 months, aged from 4 days to 14 years. Clinical presentation depends from age of diagnosis, where children diagnosed less than 3 months of age (18 of them or 29\%) manifested signs of heart failure; children diagnosed under from 3 to 12 month (32 or $51.6 \%$ ) manifested signs of cardiac failure, often respiratory infection. Diagnosis were decided by echocardiography, where peak velocity was estimated by continuous Doppler. All children age under one year, disregard of type of coarctation, therapy was surgical, end-to-end anastomosis. In 4 children age 10 to 13 years, percutaneous balloon angioplasty were performed, in different centers, and in all of them short time after intervention, re-coarctation was noted. Recoarctation was noted in 6 children.

Conclusion Isolated CoA is a CHD with excellent prognosis, especially in children under one year of age. Percutaneous balloon angioplasty is performed in adult patients but short term results are delicate and stent implantation were performed.

\section{TRANSITION FROM FOETAL TO NEONATAL LIFE: CHANGES IN CARDIAC FUNCTION ASSESSED BY SPECKLE-TRACKING TECHNIQUE}

doi:10.1136/archdischild-2012-302724.1138
U Schubert. Neonatology, Karolinska University Hospital, Karolinska Institutet Stockholm, Sweden

Objective Assessment of cardiac function by speckle tracking (2D-S) echocardiography in the transitional period from foetal to neonatal life in a healthy population.

Methods Ultrasound assessment of cardiac function of 30 healthy foetuses at the gestational age of 28 and follow-up after birth using $2-\mathrm{D}$ strain derived novel parameters such as strain (S), strain rate (SR), tissue velocities, MPI- and E/E'-index, E/A- and E'/A'-rate of both right (RV) and left ventricles (LV) and interventricular septum (IVS) and comparison to conventionally measured cardiac stroke volume (SV), cardiac output (CO) and ejection fraction (EF).

Results Ultrasound performance and analysis is technically feasible in all 30 foetuses and in the neonatal period. In foetuses, tissue velocities and SR measurements are homogenous for all regions of interest in both ventricles, and strain increases from apex to basis and is higher in the RV compared to LV. All calculated indices are almost identical for RV and LV.

After birth, strain and strain rate exhibit significantly lower values $(p<0.001)$ and systolic tissue velocities are significantly $(p=0.001)$ higher in comparison to fetal values in both chambers and in all regions of interest. The conventional methods for measuring EF, SV and $\mathrm{CO}$ show higher variability and lower reproducibility.

Conclusion The haemodynamic changes in cardiac function from foetal to neonatal life can be assessed by the novel method of speckle-tracking echocardiography which seems to be more reliable than conventional ultrasound techniques. Therefore, we recommend using speckle-tracking technique in routine follow-up of myocardial function in foetuses and neonates.

\section{RIGHT HEART DIMENSIONS AND FUNCTION AT 6 YEARS- OF-AGE USING 2D-AND 3D-ECHOCARDIOGRAPHY}

doi:10.1136/archdischild-2012-302724.1139

'LA Mohlkert, ${ }^{2} \mathrm{CP}$ Halvorsen, ${ }^{3} \mathrm{G}$ Sjöberg, ${ }^{2} \mathrm{~J}$ Hallberg, ${ }^{1} \mathrm{M}$ Norman. ${ }^{1} \mathrm{Clinical}$ Science, Intervention and Technology: ${ }^{2}$ Clinical Science and Education; ${ }^{3}$ Women's and Children's Health, Karolinska Institutet, Stockholm, Sweden

Background and Aim Survival after premature birth and neonatal lung disease is nowadays almost universal. To determine the long-term health outcomes for these infants, cardio-respiratory follow-up is needed. Whereas normative reference data for lung function in children exists, reference data of cardiac structure and function are at large lacking. The aim of this study was to determine right heart dimensions, volumes and function in healthy preschool children using echocardiography. We also wanted to compare right heart dimensions from 2D-images with volumes obtained from 3D-full volume single-beat echocardiographic measurements. Methods Forty-one healthy children (23 boys) aged 6 years, mean weight $=24.1 \pm 4.3 \mathrm{~kg}$ and mean height $=121.9 \pm 4.3 \mathrm{~cm}$, were assessed with echocardiography (Acuson SC2000, Siemens). Using 2D-echocardiography, we measured right ventricular (RVmajor/ minor) and right atrial diameters (RA major/minor). Using pulsed waved Doppler tissue imaging we calculated the E/e'-ratio and the Tei-index. With 3D-echocardiography we also calculated volumes for the right ventricle (RVEDV, RVESV) and atrium (RAEDV, RAESV), as well as ejection fraction (EF) for RV.

Results The RVmaj was $53.0 \pm 3.7 \mathrm{~mm}$, RVmin $29.9 \pm 3.1 \mathrm{~mm}$, RVEDV $42.8 \pm 7.5 \mathrm{ml}$, RVESV $18.9 \pm 3.4 \mathrm{ml}$, RAmaj $35.3 \pm 3.6 \mathrm{~mm}$, RAmin $32.0 \pm 3.1 \mathrm{~mm}$, RAEDV $6.5 \pm 2.4 \mathrm{ml}$, RAESV $15.8 \pm 4.3 \mathrm{ml}, \mathrm{E} / \mathrm{e}^{\prime}$ ratio 3.3 \pm 0.7 , RV Tei-index $0.36 \pm 0.09$ and RVEF 56 (range51-61) \%. The length of right ventricle ( $R V_{\text {maj}}$ ) measured with $2 \mathrm{D}$ correlated significantly with 3D estimated volumes (RVEDV, $\mathrm{r}=0.45, \mathrm{p}<0.01$ ). Right heart volumes correlated positively with BMI; RVEDV $(r=0.61$, $\mathrm{p}<0.001), \operatorname{RVESV}(\mathrm{r}=0.62, \mathrm{p}<0.001), \operatorname{RAEDV}(\mathrm{r}=0.72, \mathrm{p}<0.001)$ and $\operatorname{RAESV}(r=0.48, \mathrm{p}<0.01)$. 
Conclusion These reference data on right ventricular and atrial dimensions and volumes can be used for follow-up studies of boys and girls at 6-years-of-age. 3D volumes correlated moderately with BMI.

\section{EFFECT OF A PILOT INTERVENTION ON SETTING UP LONG-TERM MECHANISM OF NEONATAL RESUSCITATION TRAINING IN 4 COUNTIES IN CHINA}

doi:10.1136/archdischild-2012-302724.1140

${ }^{1} \mathrm{~T} \mathrm{Xu},{ }^{1} \mathrm{X}$ Jin, ${ }^{1} \mathrm{H}$ Wang, ${ }^{1} \mathrm{~L}$ Gong, ${ }^{2} \mathrm{X}$ Zheng, ${ }^{3} \mathrm{G} \mathrm{Yu},{ }^{4} \mathrm{G} \mathrm{Xu},{ }^{5} \mathrm{P}$ Zhang. ${ }^{\mathrm{T}}$ National Center for Women and Children's Health, China CDC, Beijing; ' ${ }^{2}$ Shandong Provincial Health Bureau, Jinan; ${ }^{3}$ Heilongjiang Provincial Health Bureau; ${ }^{4}$ Heilongjiang Maternal and Child Health Care Center, Haerbin; ${ }^{5}$ Shandong Maternal and Child Health Care Center, Jinan, China

Background and Aims Neonatal mortality is the leading cause of under-5 child deaths in China. The aim was to evaluate the effect of a pilot intervention on setting up long-term mechanism of neonatal resuscitation training in 4 counties in China.

Methods A neonatal resuscitation leading group was set up within each county level hospital to lead the in-hospital training, department coordination, resuscitation practices and cases audit. A random control survey was conducted in the intervention counties and 4 randomly selected control counties to evaluate the impact of the intervention. Indicators evaluated include knowledge and selfconfidence score of health providers, in-hospital regulations, changes of asphyxia incidence and mortality.

Results Over $90 \%$ of intervention hospitals had carried out neonatal resuscitation related regulations requiring that trained paediatricians participate in case discussion of high-risk delivery and onsite resuscitation, while in control hospitals less than $55 \%$ had such requirements. The average knowledge score of health providers was significantly higher in the intervention counties than the control counties (9.19 \pm 1.18 VS $8.40 \pm 1.52)$. The average self-confidence score in the two groups were $57.33 \pm 2.50$ and $54.09 \pm 8.19$ respectively. The incidence of birth asphyxia (defined as Apgar score $\leq 7$ ) decreased from $8.83 \%$ to $5.99 \%$ in the intervention counties, and the intrapartum-related deaths in the delivery room decreased from 27.60 to 5.03 per 100,000 . No significantly changes were found in the control counties.

Conclusions Setting up long-term mechanism of neonatal resuscitation training is an effective method to strengthen in-hospital training, build up capability for neonatal resuscitation and therefore, can decrease the incidence of neonatal asphyxia.

\section{THE DIAMETER OF CORONARY ARTERIES IN HEALTHY NEWBORNS AT BIRTH, ONE AND SIX MONTHS OF AGES}

doi:10.1136/archdischild-2012-302724.1141

'BS Karagol, '2UA Orun, 'A Zenciroglu, 'N Okumus, 'N Karadag, 'A Dursun, 'AA Kundak, ${ }^{2} S$ Karademir. 'Dr. Sami Ulus Maternity, Childrens' Education and Research Hospital, Division of Neonatology; 'Dr. Sami Ulus Maternity, Childrens' Education and Research Hospital, Division of Pediatric Cardiology, Ankara, Turkey

Objective The aim of this study was to determine accurate and validated $z$-score equations for the normal values of coronary diameters adjusted with growth changes of the neonates by testing three different time periods.

Methods Echocardiography was performed on 200 healthy neonates at birth, one and six months of ages. Several regression models for the left (LCA) and right coronary artery (RCA) diameters were tested with weight, height, body surface area and aortic annulus diameter. Reliable standards for the coronary artery diameters for healthy newborns by determining z-score equations with appropriate statistical validations were established.

Results The coronary diameters on birth measurements were strongly correlated with birth weight, height, surface area and the diameter of the aortic annulus (Pearson's $\mathrm{R}>0.8$, all $\mathrm{p}<0.01$ ). There were significant associations between the mean data of weight, height, body surface area, aortic annulus and the LCA and RCA diameters measurements of study subjects at birth, one and six months of ages $(p<0.05)$

Conclusion We present a new set of equations for neonatal z-score calculation on the basis of a large number of healthy neonates on three different time period consisting of birth, one and six month of ages. It is clear with this study that the growth in caliber of the coronary arteries is definite and progressive during postnatal time.

\section{SAFEGUARDING THE BRAINS OF OUR SMALLEST CHILDREN: SAFEBOOSC - THE PILOT STUDY}

doi:10.1136/archdischild-2012-302724.1142

${ }^{1} \mathrm{~S}$ Hyttel-Sørensen, ${ }^{2} \mathrm{~T}$ Austin, ${ }^{3} \mathrm{M}$ Benders, ${ }^{4} \mathrm{O}$ Claris, ${ }^{5} \mathrm{G}$ Dempsey, ${ }^{6} \mathrm{M}$ Fumagalli, ${ }^{7} \mathrm{C}$ Gluud, ${ }^{8} \mathrm{C}$ Hagmann, ${ }^{9} \mathrm{~L}$ Hellström-Westas, ${ }^{3 P}$ Lemmers, ${ }^{10} \mathrm{G}$ Naulaers, ${ }^{11} \mathrm{~A}$ Pellicer, ${ }^{12} \mathrm{G}$ Pichler, ${ }^{7} \mathrm{~L}$ Saem Støy, ${ }^{3} \mathrm{~F}$ van Bel, ${ }^{13} \mathrm{~W}$ van Oeveren, ${ }^{14} \mathrm{M}$ Wolf, ${ }^{15} \mathrm{G}$ Greisen. ${ }^{1}$ Copenhagen University Hospital Rigshospitalet, Copenhagen, Denmark; ${ }^{2}$ Rosie Maternity Hospital, Department of Paediatrics, Cambridge, UK; ' Wilhelmina Children's Hospital, Department of Neonatology, Utrecht, The Netherlands; ${ }^{4}$ Hospices Civils de Lyon, Department of Neonatology, Lyon, France; ${ }^{5}$ University College Cork, Department of Neonatology, Cork, Ireland; ' Università di Milano, Department of Maternal and Pediatric Sciences, Milan, Italy; ' Copenhagen Trial Unit, Rigshospitalet, Copenhagen, Denmark; ${ }^{8}$ University Hospital Zurich, Clinic of Neonatology, Zurich, Switzerland ${ }^{9}$ University Hospital Uppsala, Department of Neonatology, Uppsala, Sweden; ${ }^{10}$ Katholieke Universiteit Leuven, Department of Neonatology, Leuven, Belgium; ${ }^{11}$ La Paz University Hospital, Department of Neonatology, Madrid, Spain; ${ }^{12}$ Medical University of Graz, Department of Paediatrics, Graz, Austria; ${ }^{13}$ Haemoscan B.V., Groningen, The Netherlands; ${ }^{14}$ University Hospital Zurich, Biomedical Optics Research Laboratory, Zurich, Switzerland; ${ }^{15}$ Copenhagen University Hospital, Rigshospitalet, Copenhagen, Denmark

Background and Aims The SafeBoosC project aims to test the benefits and harms of cerebral near-infrared spectroscopy (NIRS) oximetry in infants born before 28 weeks of gestation. A phase II trial will randomise infants to cerebral NIRS oximetry and a treatment guideline during the first 72 hours of life or standard care with blinded NIRS-monitoring. The primary outcome is the area (burden) outside the normal ranges of $\mathrm{rStO}_{2}$ of 55 to $85 \%$.

Methods This pilot of the experimental group included 10 infants using the INVOS 5100C and NONIN EQUANOX 7600.

Results Median gestational age was 26 weeks +3 days. Median start-up time was 133 minutes after delivery. Median recording time was 69.7 hours. Mean $\mathrm{rStO}_{2}$ was $64.2+/-4.5 \%$. Median burden of hyper- and hypoxia was $30.3 \%$ hours (range 2.8-112.3). Clinical staff responded to out of range values 29 times - once was it to a value above $85 \%$. In comparison, there was 83 periods of more than 10 minutes with $\mathrm{rStO}_{2}$ below $55 \%$ and four episodes with $\mathrm{rStO}_{2}$ above $85 \%$. These periods accounted for $72 \%$ of the total burden of hypoxia. 18/29 of interventions were adjustments of $\mathrm{FiO}_{2}$ that 13 of 18 times resulted in an out of range $\mathrm{SpO}_{2}$. Two infants suffered burns.

Conclusions Early cerebral NIRS monitoring proved feasible, but prolonged periods of hypoxia went untreated, while adjustment of $\mathrm{FiO}_{2}$ often would result in $\mathrm{SpO}_{2}$ out of local target range. This points to a less than perfect implementation of the treatment guideline.

\section{PREDICTORS OF DUCT DEPENDENT CONGENITAL HEART DISEASE IN INFANTS TRANSFERRED BY NEWBORN EMERGENCY TRANSPORT SERVICE (NETS) VICTORIA}

doi:10.1136/archdischild-2012-302724.1143

${ }^{1,2} \mathrm{~N}$ Gupta, ${ }^{2,3} \mathrm{COF}$ Kamlin, ${ }^{2,4} \mathrm{M}$ Stewart, ${ }^{5} \mathrm{M}$ Cheung, ${ }^{4} \mathrm{~N}$ Patel. ${ }^{1}$ Neonatal Unit, John Radcliffe Hospital, Oxford, UK; ${ }^{2}$ Newborn Emergency Transport Services (NETS), Royal Children Hospital; ${ }^{3}$ Department of Newborn Research, Royal Womens Hospital, 